\title{
artigo
}

Aguair, C.A.S.; Barbosa e Silva, M.C.; Queiroz, S.A.; Santos, R.L.;

Modelo de promoção da saúde como aporte na prática de enfermagem

DOI: https://doi.org/10.36489/saudecoletiva.2021v11i64p5604-5615

\section{Modelo de promoção da saúde como aporte na prática de enfermagem}

Health promotion model as a support in nursing practice

Modelo de promoción de la salud como apoyo en la práctica de enfermería

\section{RESUMO}

Objetivo: Descrever o Modelo de Promoção da Saúde de Nola J. Pender como aporte à prática de enfermagem. Método: Trata-se de uma revisão narrativa da literatura com a busca sendo realizada em março de 2020 por meio do buscador Google Acadêmico utilizando a expressão "Modelo de Promoção da Saúde de Nola J. Pender" para realização da busca. Resultados: Foram utilizados um total de quatro (100\%) estudos, onde um (25\%) é do tipo artigo de revisão, dois (50\%) de natureza transversal, dois $(50 \%)$ estudos são de caráter descritivo e um (25\%) possui perfil observacional ou quantitativo. Conclusão: 0 Modelo de Promoção da Saúde proposto por Pender busca identificar padrões de vida e histórico pessoal do indivíduo e facilita o direcionamento da assistência.

DESCRITORES: Enfermagem; Promoção da Saúde; Assistência Centrada no Paciente.

\section{ABSTRACT}

Objective: To describe Nola J. Pender's Health Promotion Model as a contribution to nursing practice. Method: It is a narrative review of the literature with the search being carried out from March 2020 through the Google Scholar searcher using the expression "Nola J. Pender's Health Promotion Model" to carry out the search. Results: A total of four (100\%) studies were used, where one $(25 \%)$ is a review article, two $(50 \%)$ are cross-sectional, two $(50 \%)$ studies are descriptive and one (25\%) has an observational or quantitative profile. Conclusion: The Health Promotion Model proposed by Pender seeks to identify the individual's living standards and personal history and facilitates the direction of care.

DESCRIPTORS: Nursing; Health Promotion; Evidence-Based Nursing.

\section{RESUMEN}

Objetivo: Describir el modelo de promoción de la salud de Nola J. Pender como contribución a la práctica de la enfermería. Método: Se trata de una revisión narrativa de la literatura con la búsqueda que se realiza a partir de marzo de 2020 a través del buscador Google Scholar utilizando la expresión "Modelo de Promoción de la Salud de Nola J. Pender" para realizar la búsqueda. Resultados: Se utilizaron un total de cuatro (100\%) estudios, donde uno (25\%) es un artículo de revisión, dos (50\%) son transversales, dos (50\%) estudios son descriptivos y uno (25\%) tiene una observación. o perfil cuantitativo. Conclusión: El Modelo de Promoción de la Salud propuesto por Pender busca identificar el nivel de vida y la historia personal del individuo y facilita la dirección de la atención.

DESCRIPTORES: Enfermería; Promoción de la Salud; Enfermería Basada en la Evidencia.

RECEBIDO EM: 28/01/2021 APROVADO EM: 08/02/2021

\section{Cosmo Alexandro da Silva de Aguiar}

Estudante de Enfermagem da Universidade Regional do Cariri (URCA), Membro do Grupo de Pesquisa em Saúde Coletiva (GRUPESC), Membro do Grupo de Pesquisa em Saúde da Criança e do Adolescente (GRUPECA).

ORCID: 0000-0001-5311-6814

\section{Maria Clara Barbosa e Silva}

Estudante de Enfermagem da Universidade Regional do Cariri (URCA), Membro do Projeto de Extensão: Promoção da Saúde e Sustentabilidade em Comunidades Quilombolas.

ORCID: 0000-0002-1825-4493 


\section{Santana Alves de Queiroz}

Estudante de Enfermagem da Universidade Regional do Cariri (URCA), Membro do Projeto de Extensão: Promoção da Saúde e Sustentabilidade em Comunidades Quilombolas.

ORCID: 0000-0002-2308-6113

\section{Rosely Leyliane dos Santos}

Professora Assistente do Departamento de Enfermagem, Doutorado, Universidade Regional do Cariri (URCA), Doutora em Enfermagem pela Universidade Federal do Ceará (UFC). Mestre em Enfermagem pela URCA. Especialista em Assistência e Gestão em Saúde da Família pela Faculdade de Juazeiro do Norte (FJN). Pesquisadora do Grupo de Pesquisa em Saúde Coletiva (GRUPESC-URCA).

ORCID: 0000-0002-3908-8834

\section{INTRODUÇÃO}

A expressão "promoção da saúde" parece que foi utilizada pela primeira vez, em 1946, por Sigerist. A expressão foi utilizada para definir as quatro etapas fundamentais da medicina: promoção da saúde, prevenção de enfermidades, recuperação de pessoas doentes e reabilitação. De acordo com este autor, a promoção da saúde deveria ser a oferta de condições de vida adequadas, boas condições laborais, cultura física e educação, além de meios para que haja descanso e lazer ${ }^{(1)}$.

O conceito de promoção da saúde vincula-se diretamente ao conceito presente na Carta de Ottawa que, em 1986, 35 países se comprometeram a exercer ações de promoção da saúde visando à diminuição das desigualdades presentes na saúde. Esse compromisso objetiva assegurar, à população, direito às escolhas que favoreçam tanto o seu bem-estar quanto sua saúde, além de poderem atuar de forma ativa neste processo e na melhoria da qualidade de vida ${ }^{(2)}$.

De acordo com a Carta de Ottawa, é importante que os vários fatores condicionantes como política, socioculturais, econômicos, biológicos, comportamentais e ambientais estejam em consonância com a saúde de forma que ocorra a promoção da saúde ${ }^{(1)}$.

Em 1998/1999 o Ministério da Saúde, instituiu o projeto de Promoção da Saúde, em parceria com o Programa das Nações Unidas para o Desenvolvimento, com o objetivo de normatizar a Política Nacional de Promoção da Saúde (PNPS), cuja função seria a de propagar outras formas de elaborar políticas públicas e incentivar
A expressão

"promoção da

saúde" parece que

foi utilizada pela

primeira vez, em

1946, por Sigerist.

A expressão foi

utilizada para definir

as quatro etapas

fundamentais da

medicina: promoção

da saúde, prevenção

de enfermidades,

recuperação de

pessoas doentes e

reabilitação. parcerias entre os diversos setores para que houvesse a ampliação de debates a respeito dos determinantes sociais de saúde ${ }^{(3)}$.

A Lei Orgânica do Sistema Único de Saúde (SUS) e a Constituição Federal de 1988, já possuírem em sua essência, normas que remetiam à promoção da saúde. A PNPS foi efetivada no ano de 2006, ao ser avaliada e aprovada pela Comissão Integradora Tripartite (CIT) e, em 2014, pelo Comitê Nacional de Saúde, ao ser reconhecido o impacto que os determinantes sociais da saúde possuem ao processo de saúde-doença, além de considerar a interdisciplinaridade e a integração entre os vários setores para a ocorrência de melhoria das condições de vida ${ }^{(2)}$. Neste contexto, foram elaboradas inúmeras estratégias e teorias que têm como objetivo a promoção da saúde da população assistida nos serviços assistenciais.

Quando se trata do Modelo de Promoção de Saúde de Nola J. Pender, ainda há necessidade de se ampliar esta discussão. Apesar de estudos utilizando o Modelo de Promoção da Saúde de Nola J. Pender já terem sido realizados em diversos contextos e culturas, além de também haver estudos com diversas faixas etárias, em vários países e, em diferentes contextos ${ }^{(4-7)}$; é importante elucidar para os(as) enfermeiros(as) sobre as teorias e os modelos de promoção da saúde desenvolvidos para que os mesmos sejam utilizados na prática clínica de forma a evitar agravos a saúde da população, além de fundamentar ainda mais a prática profissional. Diante disso, este estudo teve como principal objetivo descrever o Modelo de Promoção da Saúde de Nola J. Pender como aporte à prática de enfermagem. 


\section{artigo}

Aguair, C.A.S.; Barbosa e Silva, M.C.; Queiroz, S.A.; Santos, R.L.;

Modelo de promoção da saúde como aporte na prática de enfermagem

\section{MÉTODO}

Trata-se de uma revisão narrativa da literatura, definida como publicaçóes amplas voltadas para discutir e caracterizar um determinado tema sob uma ótica contextual e/ou teórica, constituindo uma análise realizada pelo autor utilizando uma perspectiva crítica da literatura publicada ${ }^{(8)}$.

Este estudo é voltado para a descrição do Modelo de Pender como aporte para a prática de enfermagem. Por conseguinte, a pergunta norteadora: "Qual a descrição do Modelo de Promoção da Saúde desen- volvido por Nola J. Pender como aporte à prática de enfermagem?".

A busca foi realizada no período de março de 2020 por meio do buscador Google Acadêmico utilizando a expressão "Modelo de Promoção da Saúde de Nola J. Pender" para realizar a busca. Os critérios de inclusão foram estudos publicados nos últimos dez anos, nos idiomas português, inglês e espanhol, disponíveis para leitura na íntegra e/ou para download.

Foi feita uma análise crítica dos estudos selecionados, de modo a ser possível a realização da síntese dos principais achados contidos nos estudos, em que foram uti- lizados os aspectos presentes no modelo de Pender apresentados e discutidos nos estudos.

\section{RESULTADOS}

Os estudos selecionados para compor a amostra do estudo encontram-se sumarizados na tabela 1. Foram utilizados um total de quatro (100\%) estudos, onde um $(25 \%)$ é do tipo artigo de revisão, dois $(50 \%)$ de natureza transversal, dois (50\%) estudos são de caráter descritivo e um $(25 \%)$ possui perfil observacional ou quantitativo.

\section{Tabela 1. Sumarização dos estudos selecionados. Crato - CE, Brasil, 2021.}

\section{Autor(es)}

Objetivo(s)/ Ano de Publicação

Hoyos GPA;

Borjas DMB;

Ramos AS;

Meléndez

$\mathrm{RMO}^{(9)}$.

Alencar

$T D^{(10)}$.

Rahimian $\mathrm{H}_{\text {, }}$ Mohammadi M,

Mehri A, Rakhshani $\mathrm{MH}^{(7)}$.
Analisar artigos originais publicados em no período de 2000-2010, identificando os principais aspectos e a forma como o modelo de Pender foi analisado. 2011
Analisar a consulta e os instrumentos de enfermagem empregados para promoção da saúde em idosos frequentadores de grupo de terceira idade; Descrever o perfil de adesão e autoeficácia dos clientes; Identificar diagnósticos e intervenções de promoção da saúde e discutir a aplicação dos instrumentos baseando-se no modelo de Pender. 2014

\section{Examinar o impacto da realização} do MPS na atividade física de voluntários saudáveis. 2016
Tipo de Estudo

Artigo de revisão.

esquisa quantitativa de caráter observacional, descritiva e transversal.
Estudo transversal iniciado em 2015 como parte de um plano intervencionista de três meses realizado com 80 voluntários, onde foram selecionados por meio um método de uma amostragem aleatória de múltiplas etapas e divididos em grupo controle e de intervenção. Foi realizado coleta de dados por meio de dois questionários: o demográfico e a versão persa do questionário internacional de atividade física.
Participaram 31 idosos. Com faixa etária média de 71,29 anos, maioria são do sexo feminino, muitos eram viúvos, eutróficos, porém, grande parte apresentava obesidade abdominal, baixo controle pressórico, realizavam acompanhamento para de até duas especialidades médicas e faziam uso de polifarmácia.

Antes da realização da intervenção, os benefícios percebidos foram avaliados como sendo "bom", no entanto a autoeficácia e comportamento foram identificados como sendo 'pobres". A prática de atividade física foi percebida como tendo uma influência positiva nos benefícios percebidos, autoeficácia, emoção, influências situacionais e comprometimento, em contrapartida tem um impacto negativo nas barreiras percebidas. $66,8 \%$ da atividade física foi prevista nas variáveis do MPS de Pender. 


\section{Analisar a aplicação do Modelo de Promoção da Saúde no atendi- \\ Gama GA ${ }^{(11)}$. \\ Processo de Enfermagem. \\ 2017}

Estudo qualitativo com abordagem descritiva, realizado com 16 gestante em pré-natal de risco habitual, com a coleta realizada durante as consultas seguindo roteiro semiestruturado.

A faixa etária predominante foi dos 20 aos 25 anos de idade, as gestantes possuíam peso adequado, ensino médio completo, união estável, estavam desempregadas, em gravidez subsequente e não planejada. Os comportamentos pregressos estavam relacionados a prática de atividade física, alimentação e nutrição e exposição ao fumo. Os benefícios percebidos foram os relacionados ao feto e ao alívio dos desconfortos presentes na gestação. As barreiras foram a rotina sobrecarga, os desejos alimentares e a indisposição. Grande parte das gestantes participantes mostraram-se capazes de modificar seus comportamentos e condutas para aqueles que promovem a saúde.

\section{DISCUSSÃO}

O Modelo de Promoção da Saúde (MPS) proposto por Nola J. Pender, pode nortear o Processo de Enfermagem, com base na identificação de fatores pregressos que tenham influência direta no comportamento de saúde do paciente; tendo como foco do modelo as crenças que podem ser avaliadas pelo enfermeiro e que são pontos críticos da intervenção deste profissional, no qual ambos, enfermeiro e cliente, devem trabalhar juntos para que um comportamento saudável seja alcançado ${ }^{(12)}$.

Nola J. Pender nasceu na cidade de Michigan, nos Estados Unidos em 1941. Aos sete anos de idade, decidiu seguir a carreira de enfermagem ao presenciar sua tia receber cuidados de uma enfermeira. Parece que mantinha a ideia de que a enfermagem deveria levar, às pessoas, ao cuidado delas mesmas ${ }^{(13)}$.

Pender parece que assumiu uma postura focada na promoção da saúde, bem como na prevenção de enfermidades, com a visão de que é preferível vivenciar a experiência do bem-estar concomitante ao ato de prevenir doenças, ao invés de deixar que uma enfermidade prevenível se instale, para que só depois seja dado o início o seu tratamento ${ }^{(13)}$.

Em 1982, Pender publicou seu modelo teórico pela primeira vez, intitulado Health Promotion in Nursing Practi-

\section{Pender parece}

que assumiu uma

postura focada

na promoção da

saúde, bem como

na prevenção de enfermidades, com

a visão de que é

preferível vivenciar a

experiência do bem-

estar concomitante

ao ato de prevenir

doenças... ce, utilizando como embasamento para sua construção três (03) conceitos fundamentais: a Enfermagem Holística, a Teoria da Aprendizagem e a Psicologia Social. Além disso, o Modelo da Valorização da Motivação Humana também foi utilizado com fundamento para a sua teoria ${ }^{(11)}$.

O objetivo desta integração, entre diversas teorias e modelos assistenciais, foi devida a busca da interpretação das relações existentes entre os estilos de vida favoráveis à saúde e os comportamentos adotados que o sujeito adere em seu contexto biopsicossocial ${ }^{(4)}$. A estrutura do modelo é construída de forma que o enfermeiro possa ofertar assistência de forma individualizada ou reunindo um grupo de indivíduos, fazendo com que o planejamento, a intervenção e a avaliação das ações realizadas seja possível ${ }^{(14)}$.

Os benefícios deste modelo ocorrem devido ao fato que ele não se limita a somente duas ou três variáveis explicativas, além de ter como vantagens percebidas a atividade, a percepção das barreiras para a atividade, a autoeficáfica para a realização da atividade. Ademais, possuí dois fatores relacionados às influências interpessoais e as situacionais, sendo que todos estes fatores são preditivos para o desenvolvimento da promoção da saúde $e^{(7)}$.

Além disso, o modelo de promoção da saúde de Pender também auxilia na identificação do modo como as pessoas 


\section{artigo}

Aguair, C.A.S.; Barbosa e Silva, M.C.; Queiroz, S.A.; Santos, R.L.;S

alteram seu comportamento com o intuito de melhorar seu bem-estar e hábitos de vida, bem como a si mesmos ao identificar os fatores prejudiciais de saúde ao mesmo tempo em que promove ações de prevenção de doenças ${ }^{(15)}$.

Outro aspecto importante do modelo de Pender é o fato dele ser aplicável em diversas populações e em diferentes contextos, constituindo algo de importante na aplicação das intervenções de enfermagem, no âmbito tanto da saúde coletiva quanto na saúde individual ${ }^{(11)}$. Além disso, o MPS possui caráter multidimensional já que leva em consideração a interação entre os fatores individuais e ambientais $^{(6)}$.

O Modelo de Promoção da Saúde de Nola J. Pender é constituído por três componentes, visando avaliar o comportamento individual que leva as pessoas a buscar a promoção da saúde: 1 - as características e experiências individuais (constituído por fatores pessoais associados a comportamentos anteriores); 2 - os sentimentos e conhecimentos sobre o comportamento que se deseja alcançar (que inclui barreiras, autoeficácia, percepção de benefícios e influências inter- pessoais) e, por último, 3 - o comportamento de promoção da saúde almejável (que abrange aspectos como compromisso com o plano de ação adotado, preferências e exigências) $)^{(14,6)}$.

A primeira esfera do modelo de Pender diz respeito aos aspectos relacionados às características e comportamentos anteriores da pessoa. Além de abranger também os fatores individuais do sujeito do cuidado, na qual estão inseridos os fatores: Biológicos, como idade e índice de massa corporal (IMC); Socioculturais, como escolaridade, etnia, nível socioeconômico, religião e estado civil; Psicológicos, como autoestima e automotivação ${ }^{(4)}$.

O comportamento anterior pode ser definido como os costumes anteriores individuais referentes à busca de saúde associada com o conceito que cada pessoa tem a respeito dos fatores e características, bem como das experiências que têm influência direta em seu estilo de vida ${ }^{(6)}$. Esta etapa faz alusão aos fatores que podem ter efeitos diretos ou indiretos no comprometimento na realização da atividade de promoção da saúde ${ }^{(9)}$.

A segunda etapa do modelo diz

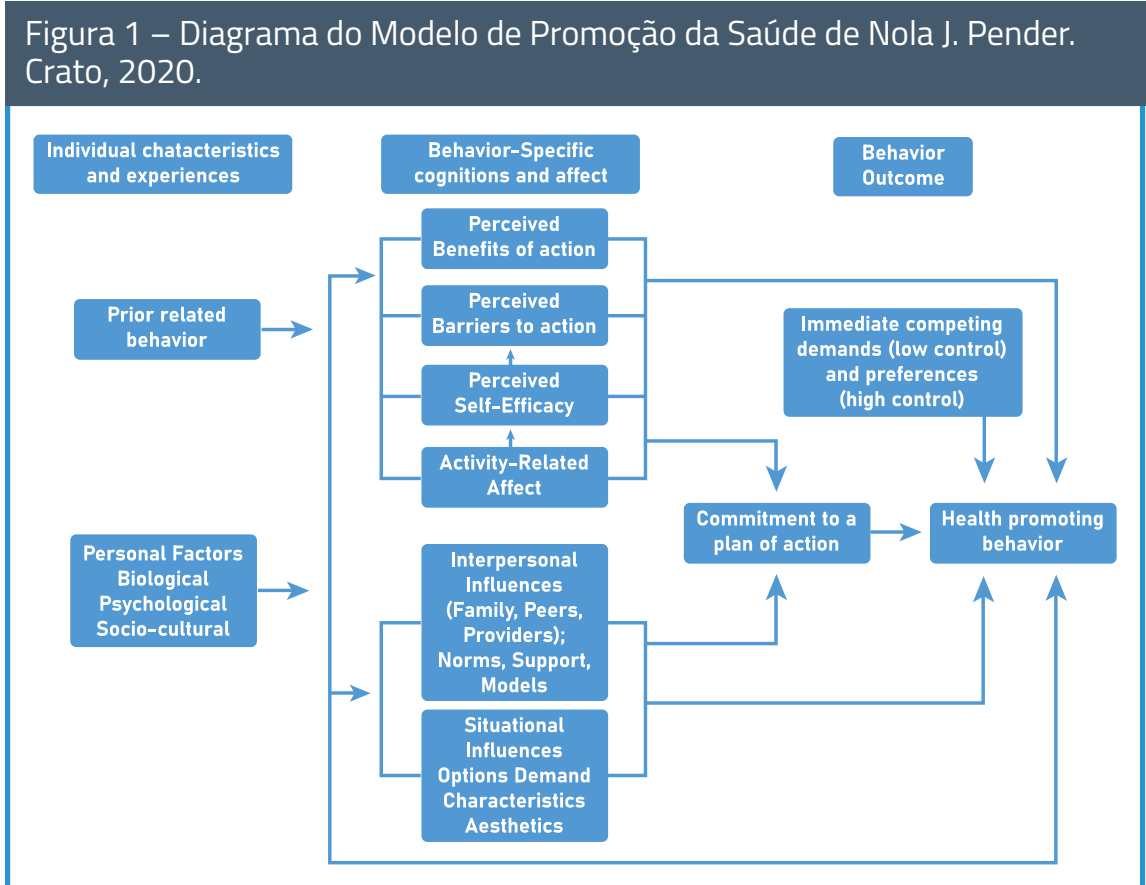

Resived Health Promotion Model. Fonte ${ }^{16}$. respeito à percepção de benefícios do comportamento (resultados positivos), como também às barreiras para a adoção de ações de promoção (pensamentos negativos sobre as dificuldades para se adquirir tais ações), influências tanto interpessoais (relativa a outras pessoas, comportamentos e crenças e de apoio social) quanto situacionais (relação entre hábitos de vida e meio ambiente), autoeficácia (capacidade de executar ações de promoção da saúde) e sobre os sentimentos relacionado ao comportamento (podendo ser positivos ou negativos, subjetivos ou objetivos, até mesmo referentes ao ato antes, durante ou depois da ação promotora de saúde) ${ }^{(11)}$.

Os conceitos pertencentes ao segundo processo do modelo aludem aos sentimentos e afetos referentes à conduta individual, sendo que as percepções dos benefícios das ações referem-se aos resultados positivos que serão alcançados com a execução da prática de promoção da saúde. As barreiras percebidas aludem as desvantagens que o indivíduo possui e que podem servir como obstáculo para a execução da ação de saúde. A percepção da autoeficácia concerne à compreensão da pessoa em realizar a ação e a eficácia percebida pelo cliente durante e após a adoção/ execução do plano de cuidados, o sentimento relacionado ao comportamento que é definido como as emoções favoráveis ou não com a conduta adotada ${ }^{(9)}$.

As influências interpessoais são aquelas nas quais as pessoas mais próximas dos indivíduos oferecem apoio e suporte para que os pacientes executem ações de saúde. Por último, as influências situacionais aludem às condições espaciais que podem influenciar de forma positiva ou negativa a realização da atividade promotora de saúde ${ }^{(9)}$.

O último componente do modelo é referente ao compromisso do indivíduo quanto ao plano de ação (propósito de elaborar meios que facilitem a execução do comportamento), às demandas competitivas imediatas (atividades que escapam do controle do sujeito como tra- 
balho e responsabilidades com a família) e preferências (comportamentos relacionados às atividades promotoras de saúde com as quais o indivíduo já detém maior controle como as questôes voltadas para a alimentação) e ao comportamento de promoção da saúde (aquisição de resultados positivos, como realização pessoal e/ ou bem-estar) ${ }^{(11)}$.

Esta etapa remete ao sucesso da adoção e execução da conduta promotora de saúde quando há o compromisso com a ação, quando não há um obstáculo inevitável ou uma preferência que seja contrária à promoção da saúde ${ }^{(10)}$. Os componentes desta etapa consistem nos conceitos pre- liminares para que o resultado final esperado seja alcançado ${ }^{(9)}$.

Com isso, o principal objetivo do MPS é assistir os profissionais de enfermagem no entendimento dos principais determinantes comportamentais de saúde como norte para aconselhar uma mudança de comportamento, resultando na promoção um estilo de vida mais saudável ${ }^{(13)}$. $\mathrm{O}$ modelo de Pender tem o intuito de unir os conceitos de comportamento com as ações voltadas para o desenvolvimento de ações que estimulam o emprego de novos estilos de vida saudáveis ${ }^{(17)}$.

Como limitação do estudo percebeu-se que a utilização de apenas três idiomas de escolha. Sendo que estudos de outras línguas, como japonês, por exemplo, poderiam ter ampliado outras contribuiçôes para a construção do vigente estudo.

\section{CONCLUSÃO}

Percebe-se que o Modelo de Promoção da Saúde proposto por Pender busca identificar os padrões de vida e histórico pessoal do indivíduo. O MPS pode oferecer um suporte à prática de enfermagem, ao auxiliar a construção do plano de cuidados para que haja a promoção da saúde e participação ativa do indivíduo em seu processo de cuidado.

\section{REFERÊNCIAS}

1. Machado TG. Promoção da saúde no programa de saúde da família: uma proposta de intervenção. [Trabalho de Conclusão de Curso]. Juiz de Fora: Universidade Federal de Minas Gerais, Curso de Especialização em Estratégia de Saúde da Família. 2015.

2. Malta DC, Reis AAC, Jaime PC, Morais Neto OL, Silva MMA, Akerman M. O SUS e a Política Nacional de Promoção da Saúde: perspectiva resultados, avanços e desafios em tempos de crise. Rev. Ciência \& Saúde Coletiva. 2018; 23(6): 1799-1809.

3. Malta DC, Morais Neto OL, Silva MMA, Rocha D, Castro AM, Reis AAC et al. Política Nacional de Promoção da Saúde (PNPS): capítulos de uma caminhada ainda em construção. Rev. Ciência \& Saúde Coletiva. 2016; 21(6): 1683-1694.

4. Bessa CC. Silva LA, Sousa TM, Silva VM, Galvão MTG, Guedes NG. Controle de saúde de celíacos: análise segundo o modelo de promoção da saúde de Pender. Texto Contexto Enferm. 2020; 29:1-11.

5. Silva ACC, Santos I. Promoção do autocuidado de idosos para o envelhecer saudável: aplicação da teoria de Nola Pender. Texto Contexto Enferm. Florianópolis. 2010 out-dez; 19(4): 745-53.

6. Guedes NG, Moreira RP, Cavalcanti TF, Araujo TL, Ximenes LB. Atividade física de escolares: análise segundo o modelo teórico de promoção da saúde de Pender. Rev. Esc. Enferm USP. 2009; 43(4): 774-780.

7. Rahimian M, Mohammadi M, Mehri A, Rakhshani MH. Impact of Performing Health Promotion Model Intervention on Physical Activity of Health Volunteer of Torbat-e-Jam City, Iran. Quarterly of International Archives of Health Sciences. 2016; 3(3): 87-91.

8. Rother ET. Revisão Sistemática x Revisão Narrativa [editorial]. Acta Paul. Enferm. 2007; 20(2):1-2.

9. Hoyos GPA, Borjas DMB, Ramos AS, Meléndez RMO. El modelo de promoción de la salud de Nola Pender: Una reflexión en torno a su comprensión. Enfermería Universitaria ENEO-UNAM. 2011 oct.-dic; 8(4): 16-23.
10. Alencar TD. A consulta de enfermagem fundamentada no "modelo de promoção da saúde de nola pender" a uma população idosa: estudo transversal [Trabalho de Conclusão de Curso]. Niterói, Rio de Janeiro: Universidade Federal Fluminense. 2014.

11. Gama GA. Modelo de promoção da saúde de Nola Pender na consulta de enfermagem à gestante [Dissertação]. Maceió: Universidade Federal de Alagoas, Programa de Pós-Graduação em Enfermagem. 2017.

12. Pender NJ. Health Promotion Model Manual. 2011. [online] Available from: < https://deepblue.lib.umich.edu/bitstream/handle/2027.42/85350/HEALTH_PROMOTION_MANUAL_Rev_52011.pdf?sequence=1\&isAllowed=y $>$ Acesso em: 15/03/2020 às $16: 36 h$

13. Nicolau IR. Atuação do enfermeiro em equipe multiprofissional no cuidado a obesos grau III. [Dissertação]. Niterói, Rio de Janeiro: Universidade Federal Fluminense, Escola de Enfermagem Aurora de Afonso Costa, Mestrado Profissional Enfermagem Assistencial. 2015.

14. Victor JF, Lopes MVO, Ximenes LB. Análise do diagrama do modelo de promoção da saúde de Nola J. Pender. Acta Paul Enferm. 2005; 18(3): 235-240.

15. Bermudez EAZ, Rodriguez YS, Sequeira ODT. Factores modificantes de la salud según el modelo de Nola j pender que influyen en una amenaza de parto prematuro en las gestantes adolecentes que asiste al puesto de salud [Seminario]. Nicaragua: Universidad Nacional Autónoma de Nicaragua. 2016.

16. Pender NJ, Murdaugh CL, Parsons MA. Health Promotion in Nursing Practice. $7^{\text {a }}$ ed. Boston, MA: Pearson; 2015

17. Guimarães HC, Borges MS, Souza M, Ribeiro MS. A Promoção da Saúde dos Portadores de HIV/AIDS em Situação Prisional Aplicado ao Modelo de Nola Pender: Estudo Qualitativo. Investigação Qualitativa em Saúde. 2017; 2: 421-430. 